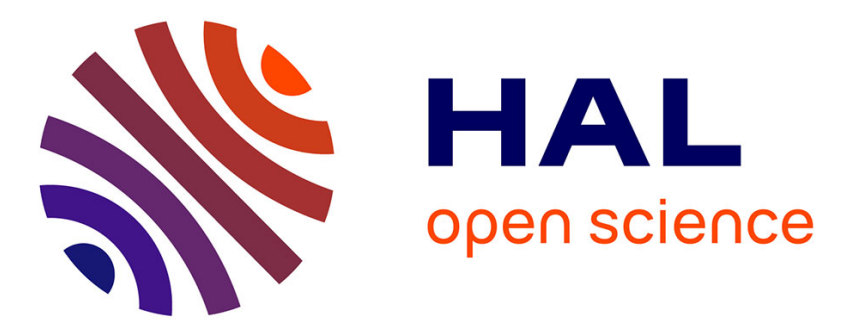

\title{
Les données sur la nature entre rationalisation et passion
} Pierre Alphandéry, Agnès Fortier

\section{To cite this version:}

Pierre Alphandéry, Agnès Fortier. Les données sur la nature entre rationalisation et passion. Revue du MAUSS, 2013, 2 (42), pp.202-220. 10.3917/rdm.042.0202 . hal-01198192

\section{HAL Id: hal-01198192 \\ https://hal.science/hal-01198192}

Submitted on 28 May 2020

HAL is a multi-disciplinary open access archive for the deposit and dissemination of scientific research documents, whether they are published or not. The documents may come from teaching and research institutions in France or abroad, or from public or private research centers.
L'archive ouverte pluridisciplinaire HAL, est destinée au dépôt et à la diffusion de documents scientifiques de niveau recherche, publiés ou non, émanant des établissements d'enseignement et de recherche français ou étrangers, des laboratoires publics ou privés. 


\section{LES DONNÉES SUR LA NATURE ENTRE RATIONALISATION ET PASSION}

Pierre Alphandéry et Agnès Fortier

La Découverte | Revue du MAUSS

$2013 / 2-n^{\circ} 42$
pages 202 à 220

ISSN 1247-4819

Article disponible en ligne à l'adresse:

http://www.cairn.info/revue-du-mauss-2013-2-page-202.htm

Pour citer cet article :

Alphandéry Pierre et Fortier Agnès, « Les données sur la nature entre rationalisation et passion », Revue du MAUSS, 2013/2 n 42, p. 202-220. DOI : 10.3917/rdm.042.0202

Distribution électronique Cairn.info pour La Découverte.

(C) La Découverte. Tous droits réservés pour tous pays.

La reproduction ou représentation de cet article, notamment par photocopie, n'est autorisée que dans les limites des conditions générales d'utilisation du site ou, le cas échéant, des conditions générales de la licence souscrite par votre établissement. Toute autre reproduction ou représentation, en tout ou partie, sous quelque forme et de quelque manière que ce soit, est interdite sauf accord préalable et écrit de l'éditeur, en dehors des cas prévus par la législation en vigueur en France. II est précisé que son stockage dans une base de données est également interdit. 


\title{
Les données sur la nature entre rationalisation et passion
}

\author{
Pierre Alphandéry et Agnès Fortier
}

\section{Introduction}

La nature est aujourd'hui inséparable d'équipements qui l'auscultent, la découpent en composantes, la quantifient, la qualifient, la mettent en réseau, etc. Au nom de « la défense du vivant » se déploient des investigations scientifiques, la production de données naturalistes et la mise en place de banques de données. À y regarder de plus près cependant, cette vision dominante d'une nature rationalisée et objectivée a cohabité et cohabite toujours avec des visions plus subjectives.

L'apparition des politiques de protection de la nature en France s'effectue au cours des décennies 1960 et 1970 . Elle s'accompagne de la mise en place d'un édifice institutionnel : création des Parcs nationaux (1960), des Parcs naturels régionaux (1967), du ministère de l'Environnement (1971), et par la loi du 10 juillet 1976 qui incarne les fondements politico-juridiques de la protection de la nature. Avec la construction de cet édifice, destiné à gérer les espaces dotés d'une nature « remarquable », s'opère un « grand partage » avec le reste du territoire national qui demeure voué à une agriculture intensive possédant ses propres institutions 
encadrant les exploitations et contrôlant l'usage du foncier ${ }^{1}$. Le développement de préoccupations liées à la nature ne se traduit pas, sauf dans le territoire des Parcs nationaux, par la constitution d'un corps de spécialistes. L'article 1 de la loi de 1976 stipule qu' « il est du devoir de chacun de veiller à la sauvegarde du patrimoine naturel dans lequel il vit », ouvrant ainsi, à tous ceux qui le souhaitent, un cadre d'engagement en faveur de la nature au nom de l' « intérêt général ». Sur le terrain, les actions menées reposent largement sur les associations naturalistes qui connaissent un essor important durant les décennies 1970 et 1980 . Regroupant des scientifiques et des amateurs, ces structures intermédiaires entre les individus et le collectif ont initié des activités de vigilance, d'alerte, de publicisation des problèmes et ont été incitées par les pouvoirs publics à développer leurs compétences scientifiques et gestionnaires. La convocation de la science au chevet de la nature a suscité la mise en place d'instruments de production et de circulation des connaissances (inventaires, études d'impact, plans de gestion de sites, constitution de réseaux d'experts) et s'est traduite par la réalisation de formes diverses de zonage de l'espace en fonction de ses qualités écologiques, conférant ainsi une visibilité à la nature. Néanmoins, l'accumulation et la mise en œuvre de ces compétences, au nom de la science, n'a pas signifié l'abandon des pratiques et des valeurs qui fondent la diversité des rapports à la nature et à sa protection dont sont porteuses les associations.

Cette diversité se fonde sur la multiplication, dans les années 1980, des courants se réclamant de l'écologie [Alphandéry et al., 1991]. On peut distinguer, au sein de cette «nébuleuse », au moins deux types de registre, souvent entremêlés. Le premier, en écho à la rhétorique alarmiste, est centré sur l'état de la planète Terre. Il se réclame d'une écologie scientifique et globale ${ }^{2}$ tout en développant, localement, des outils de gestion de la nature à des fins de reproduction des ressources naturelles. Dans cette acception,

1. Ce partage entre agriculture et nature peut n'être que partiel, ouvrant des zones d'activité communes qui vont nourrir des expérimentations ou des conflits souvent récurrents.

2. Elle-même divisée en courants (préservationniste, conservationniste, développementaliste...) qui se disputent la direction des grandes organisations internationales dédiées à l'environnement [Brahy et Louafi, 2004]. 
la nature devient une ressource quantifiable, objet de politiques publiques qui s'accompagnent de la constitution progressive d'un appareil statistique par l'État. L'autre registre articule davantage les menaces écologiques à un discours politique critique des modes de vie contemporains tout en s'interrogeant sur les manières diverses d'habiter la planète. Pour Serge Moscovici [2002], par exemple, l'enjeu n'est pas seulement de gérer la nature comme un objet dont nous serions séparés, mais de l'historiciser, de la penser comme un produit de nous-mêmes et en fonction de ce que nous voulons en faire. L'écologie politique correspond à une remise en cause de la vision dominante du progrès, des formes prescriptives et centralisées de la modernisation d'après-guerre et préconise des modes de vie plus autonomes, "plus proches de la nature », privilégiant «l'être sur l'avoir».

Le passage du modèle de la protection de la nature à celui de la conservation de la biodiversité, au début des années 1990, traduit un changement de paradigme scientifique et un changement d'échelle puisqu'il ne s'agit plus seulement de protéger la faune et la flore dans des réserves et des parcs, mais de sauvegarder les systèmes naturels présents sur l'ensemble de la planète et qui sont souvent présentés d'un point de vue utilitariste comme assurant un ensemble de services (écosystémiques) indispensables à notre survie. Comme le résume Patrick Blandin, professeur au Muséum d'histoire naturelle, « La Biodiversité a remplacé la Nature. La Protection a cédé le pas à la Gestion » [Blandin, 2009, p. 33]. La dimension scientifique et gestionnaire du discours écologique semble avoir pris le pas sur la critique et l'imagination issues de l'écologie politique, comme le suggère Pierre Lascoumes dès 1994. Avec la biodiversité, l'équipement des politiques s'intensifie. Elles requièrent une grande masse d'informations, des banques de données interopérables et accessibles à tous, imposent des normes et standardisent des procédures. Tout ceci est rendu possible par le développement des outils de traitement de l'information fondés sur les mathématiques et l'informatique qui accroissent les capacités de stockage des données et favorisent l'essor de la modélisation [Pestre, 2013 ; Bowker, 2000 ; Tsoukas, 1997]. Enfin, le poids croissant des risques globaux (biodiversité, changement climatique) et du néolibéralisme participent de l'affirmation de cette dynamique scientifique et gestionnaire. 
Toutefois, le monde associatif continue d'être le théâtre d'expressions multiples, en particulier dans le domaine de la production des données naturalistes et de la mise en place des systèmes d'information, outils indispensables pour élaborer, mettre en œuvre et évaluer les politiques de conservation de la biodiversité. Nous voudrions montrer que cette production de données relève de deux registres de rapports à la nature, apparemment contradictoires, qui mêlent d'un côté des considérations scientifiques et gestionnaires et de l'autre des rapports subjectifs fondés sur la proximité, la familiarité, les savoirs non scientifiques, etc. Cette hypothèse rejoint les observations de Marc Mormont sur l'existence de deux grandes catégories d'appréhension de la nature : une nature rationalisée et maîtrisable et une nature intuitive qui « révèle que la culture même arrimée à la science et armée de la technique comporte d'autres formes de relations à la nature que la relation utilitaire, technique qui la réduit à une ressource productive » [Mormont, 1998,p. 46]. Quels que soient les efforts de rationalisation entrepris, la production des données reste fortement marquée par les valeurs et le rapport à la nature de ceux qui en assurent la collecte [Laurence et Turnout, 2010].

Nous rappellerons tout d'abord le rôle central joué par les associations dans la production de données naturalistes. Nous montrerons alors à partir d'un exemple concret comment une association traite la question des données dont la production a partie liée avec les liens sociaux et les formes d'engagement des membres qui la constituent. Nous insisterons ensuite sur le fait que le développement des politiques de la nature s'est traduit par un processus constant de rationalisation des données dont la forme la plus aboutie est l'adoption par le ministère de l'Écologie, en 2007, du Système d'information sur la nature et les paysages (SINP). La production des données s'inscrit dans un cadre de plus en plus large et obéit à des règles établies à l'échelon international. Elle subit aussi les effets conjoints de la professionnalisation et du néolibéralisme qui sont à l'origine de changements importants dans le fonctionnement associatif. Enfin, nous conclurons en questionnant ce qui fait aujourd'hui la spécificité associative dans les rapports à la nature. 


\section{Les associations, des acteurs clés dans la production des données sur la nature}

Les données naturalistes sont une représentation simplifiée et rationalisée de la nature. En effet, la donnée naturaliste à laquelle il est fait référence ici est le produit d'une observation faite sur le terrain, comportant au minimum quatre types d'indications : la nature du taxon, le lieu, la date et le nom de l'observateur. En France, les données naturalistes disponibles sont majoritairement produites par les associations naturalistes. Héritières des sociétés savantes du XIX ${ }^{\mathrm{e}}$ siècle [Matagne, 1999], période où la science ne se distinguait pas de la protection de la nature [Raffin et Ricou, 1985], ces structures associatives ont vu le jour avec l'arrivée des scientifiques universitaires. Le recul de la systématique ${ }^{3}$ à l'Université dans le courant des années 1970, au profit de la biologie moléculaire, a laissé le champ libre aux associations [Maurin, 1997]. Schématiquement, celles-ci se caractérisent par leur intérêt pour la production de connaissances, une légitimité en matière d'expertise et leur capacité à mobiliser des réseaux de bénévoles, amateurs pour la plupart [Micoud, 2001]. L'amateur est défini ici « comme un citoyen ordinaire doté de compétences naturalistes qu'il développe et met au service d'une cause publique hors cadre professionnel, le plus souvent au sein d'une association » [Dupré et Micoud, 2007, p. 221]. Il recouvre en réalité des profils différenciés qui se situent sur un continuum allant de l' " amateur du dimanche », dont l'expérience est limitée, la pratique irrégulière et le savoir contesté, à l'amateur «éclairé » ou « chevronné », dont les compétences sont à rapprocher de celles des professionnels qui exercent leur activité dans le cadre d'un métier.

Parce qu'il y a dans les associations des gens prêts à livrer à peu de frais des données collectées par passion, les moyens alloués par les pouvoirs publics restent limités. Ainsi, de nombreux membres d'associations ont le sentiment que leur travail de production de données naturalistes n'est pas reconnu par l'État à sa juste valeur. Cela explique l'enjeu majeur que représente la mise à disposition de l'information dans l'univers naturaliste. Si l'on adopte un point de vue utilitariste que l'on rencontre fréquemment dans le monde

3. Science de la classification des êtres vivants. 
naturaliste, la réticence à livrer les données produites trouve sa source dans la volonté d'exercer une forme de pouvoir et de monnayer des informations souvent considérées comme « un trésor de guerre » [Durand-Bourlier, 2003]. À contrario, la production de connaissances ne saurait être envisagée uniquement en termes de ressource monnayable, mais elle peut être conçue, dans cet univers composé essentiellement de bénévoles, comme une richesse sur le plan du développement personnel et une activité associant des personnes au service du bien commun [Ellis et al., 2005]. Enfin, la production de données est fréquemment associée à des liens sociaux et à l'inscription dans un territoire. Afin de comprendre la manière dont coexistent ces différentes dimensions, nous nous sommes penchés sur le fonctionnement d'une association naturaliste, la Société mycologique du Nord de la France (SMNF), avec le souci d'identifier les formes d'engagement de ses adhérents (voir encadré ci-après).

\section{La Société mycologique du Nord de la France}

Fondée en 1967, la SMNF, basée à la faculté des sciences de pharmacie et de biologie de Lille, se définit comme une association scientifique régionale, à but non lucratif, regroupant des bénévoles «s'intéressant aux champignons pour les reconnaître dans la nature, se familiariser avec leurs propriétés, leur classification, leur rôle, leur protection et leur éventuelle comestibilité ». Parmi les deux centvingts adhérents, quelques dizaines d'entre eux seulement participent régulièrement aux manifestations organisées par l'association : sorties sur le terrain, conférences, séances d'initiation à la microscopie, session annuelle de formation, expositions mycologiques ouvertes au public, publications dans le bulletin de la société. À travers la collecte de données de terrain, ils concourent à l'inventaire de la fonge régionale. Projet initié en 1991, par le président de l'association d'alors, professeur et mycologue professionnel à la faculté de pharmacie, par ailleurs président de la Société mycologique de France. Les mycologues impliqués dans la réalisation de cet inventaire sont pour l'essentiel des amateurs couvrant toute la gamme des profils existants - du débutant à l'amateur éclairé puis chevronné -, encadrés de trois professionnels, universitaires. Ce sont des personnes de sexe masculin dans leur grande majorité, plutôt âgées et issues de différents milieux sociaux. L'adhésion à une association mycologique apparaît comme un moyen privilégié d'apprentissage dans une spécialité réputée plus complexe que la botanique ou l'ornithologie. 


\section{Passions mycologiques}

La mycologie, à l'image des autres sciences naturalistes, a pour caractéristique de s'appuyer sur l'observation in situ. Elle fait ainsi partie des «sciences de plein air » [Callon et al., 2001]. L'observation des champignons dans leur milieu naturel fait appel à des connaissances théoriques mais mobilise également d'importants savoirs pratiques.

«Beaucoup de choses sont dans les bouquins - constate un professionnel
de la SMNF -, mais y a un truc qui n'est pas dans les bouquins, c'est
l'intuition. Les descriptions, les clés de détermination ont beau être
très détaillées, quelquefois les différences qui sont mentionnées ont
l'air très superficielles comme ça ; en fait, elles correspondent à des
conditions de terrain qu'il n'est pas facile de transmettre par écrit. »

Le savoir-faire des mycologues repose en effet sur la faculté à mobiliser certains sens, comme le regard ou l'odorat. Dans les entretiens, il est souvent fait allusion à tel ou tel mycologue, amateur ou professionnel, doté d' " une mémoire invraisemblable des odeurs, des goûts, de l'allure du champignon » ou « d'un sens de l'observation étonnant, un coup d'œil, un nez extraordinaire ». Sachant que les compétences varient fortement d'un individu à l'autre. Un représentant de la SMNF, scientifique et professeur d'université, distingue ainsi trois profils parmi les mycologues de terrain amateurs : le «consommateur de champignon », « le curieux qui cherche à apprendre » et « une petite minorité de personnes qui étudient, qui cherchent vraiment à connaître, apprendre, progresser, à découvrir, à contribuer à des programmes d'inventaires formalisés ». Et le même responsable de pointer l'importance de la convivialité dans les sociétés mycologiques, qui constitue un motif d'adhésion majeur pour certains, avant d'ajouter :
« Chacun fait en fonction de ses objectifs et du temps qu'il a. La mycologie active, ça prend beaucoup de temps. On ne peut pas obliger les gens à devenir mycologues à plein temps. Si on a des gens qui sont intéressés à gravir tous les échelons, on est très heureux évidemment » (entretien, 2006).

L'importance du travail de terrain explique la frontière souvent ténue qui sépare les amateurs éclairés des professionnels, cette distinction étant à la source de débats animés et récurrents [Delaporte, 1984 ; Rémy, 1995]. 
La production de savoirs, qui constitue une grande partie de l'action de la SMNF, est indissociable d'une dynamique collective. Les différentes manifestations, expositions, sorties sur le terrain, sessions de formation sont autant de cadres d'interaction pour les mycologues, qu'ils soient amateurs plus ou moins avertis ou professionnels, destinés à affiner les connaissances de chacun.

«Au niveau de la SMNF - déclare le président - on essaie de faire avancer la réflexion ou la motivation des gens - sans forcément la détourner de leur motivation première qui est souvent respectable -, mais de les enrichir dans leur approche par cet aspect de travail collectif, rigueur dans la détermination, prise en compte des champignons dans leur environnement » (entretien, 2006).

En tant que lieux d'apprentissage, ces collectifs sont aussi l'occasion fournie à chaque mycologue d'afficher ses compétences et d'en évaluer les limites. Ils fonctionnent ainsi comme une mise à l'épreuve de leur savoir par le terrain et par les autres. L'affiliation à l'association va souvent de pair avec la quête de reconnaissance, que celle-ci s'exerce au sein du groupe, par le biais des pairs, ou sur des scènes extérieures (voisinage, professionnel, etc.).

La production de connaissance, en effet, est indissociable des liens de sociabilité qui existent au sein du collectif.

«Une association de bénévoles - fait remarquer un responsable d'association -, ce sont des gens qui se connaissent, des amis, des copains. On se voit, on discute, on s'engueule, donc il y a un aspect subjectif qui n'est pas scientifique mais qui est fondamental » (entretien, 2007).

Pour beaucoup d'amateurs bénévoles affiliés à la SMNF, la mycologie est avant tout une activité où le plaisir d'observer la nature, le sentiment de responsabilité à son égard, les rapports de convivialité, le sentiment d'appartenance au groupe constituent autant d'éléments déterminants dans l'investissement et le temps consentis à cette activité. Cet ensemble de paramètres : le désir de connaissances, le plaisir d'être ensemble, la quête de reconnaissance, mais aussi la mobilisation autour d'un projet commun, en l'occurrence l'inventaire de la faune à l'échelle régionale, participent à cette dynamique collective de production des savoirs au sein de l'association. Sans le travail des bénévoles qui ne comptent ni leur temps ni leur argent, les listes d'espèces fongiques régionales ne pourraient voir le jour. Réciproquement, 
ces inventaires sont l'occasion pour les amateurs d'enrichir leurs connaissances au contact des professionnels qui montrent une grande disponibilité aussi bien à l'égard des amateurs confirmés que des débutants. Cependant, la réalisation d'un tel projet repose largement sur la confiance et la capacité du président à exercer un rôle de médiation entre le monde des gestionnaires et des scientifiques, d'une part, et celui des amateurs bénévoles, d'autre part. L'un d'eux (amateur éclairé) fait ainsi remarquer :

«Ces inventaires biologiques sont devenus aussi l'alimentaire pour beaucoup de professionnels. Ça permet de récupérer des crédits [...]. Mais c'est dit, ce n'est pas caché. On ne prend pas non plus l'amateur pour le gogo qu'on piège dans un système. Ici, en tout cas, c'est très clair. »

Après avoir constaté combien la production de données par les bénévoles est liée aux relations avec la nature, aux rapports humains ainsi qu'aux valeurs qui les structurent, il nous faut à présent insister sur le processus constant de rationalisation des données qui a accompagné la mise en place des politiques de la nature aux échelles nationale, européenne et internationale avec l'émergence de la notion de biodiversité. Ce processus intervient dans un contexte de plus en plus marqué par le néolibéralisme et le développement des banques de données et des systèmes d'information. La multiplication des règles, normes et procédures en matière de production des données contribue à dessiner une représentation de plus en plus formalisée de la nature.

\section{La normalisation de la nature et le tournant néolibéral}

Le développement des politiques de la nature, à compter des années 1970, s'est traduit par une succession d'initiatives des pouvoirs publics pour rationaliser le dispositif de recueil de l'information [Alphandéry, Fortier, 2011 ; 2012]. La première intervient à l'occasion de la loi de protection de la nature du 10 juillet 1976, qui fournit un premier cadre de rationalisation des données. Elle est suivie, quelques années plus tard, par l'organisation de l'inventaire des Zones naturelles d'intérêt écologique, faunistique et floristique (ZNIEFF). Jamais la France n'avait connu d'inventaire réalisé à une aussi large échelle et jamais les données d'espèces 
n'avaient été associées aux données sur les milieux. Ce processus précurseur s'est en grande partie appuyé sur l'investissement bénévole de nombreux amateurs associatifs.

Les années 1990 marquent un véritable tournant dans l'histoire de la protection de la nature à travers l'apparition de la notion de biodiversité, qui constitue un changement de paradigme scientifique et devient dans le même temps un enjeu planétaire. Les politiques en faveur du vivant donnent lieu à la mise en place d'équipements scientifiques à l'échelle européenne, voire internationale : banques de données, instruments de mesure, nomenclatures, inventaires, indicateurs, etc. L'instauration de ces références communes, qui garantit des comparaisons entre États au sein de l'Union européenne, répond à des préoccupations comptables de plus en plus détachées des enjeux de protection définis à l'échelle des territoires. Ce processus de rationalisation des données se poursuit à travers la signature, en 1998, de la convention d'Aarhus et la promulgation de la directive Inspire, en 2007, qui rendent obligatoire la transparence des données. En France, l'élaboration du Système d'information sur la nature et les paysages (SINP), en 2007, est la traduction des engagements européens de la France en matière d'information environnementale. Ces dispositifs, qui peuvent être assimilés à un processus de normalisation des données, consistent, au nom de la rationalité scientifique, à définir des référentiels (taxonomiques, géographiques, etc.), des protocoles communs ainsi que des critères permettant d'attester de la qualité des données. Selon un chargé de mission du ministère en charge de l'environnement :

« Il n'y a pas en France de tradition de travail en commun entre organismes. Chacun a monté sa banque de donnée, son observatoire ou ses réseaux. Le basculement des années 2000 tient à la prise de conscience que l'on ne peut continuer sans coordination, car cela empêche de valoriser les données » (entretien, 2006).

Cette dynamique, qui va de pair avec l'utilisation d'outils informatiques de plus en plus sophistiqués, contraint les associations à faire appel à des salariés qualifiés et à rationaliser le travail de collecte des bénévoles. La standardisation des connaissances exige en effet de la part de ces derniers qu'ils renseignent davantage de paramètres à propos d'une même donnée, transmettent leurs observations au moyen de l'outil informatique et enfin se conforment à des protocoles de collecte souvent complexes. Autant 
de marqueurs que certains assimilent à un processus de qualification des bénévoles là où d'autres y voient des injonctions incompatibles avec l'engagement bénévole.

À travers cette volonté de rationalisation incarnée par le SINP s'institue une autre conception de la donnée que celle qui a longtemps prévalu dans la communauté naturaliste [Alphandéry, Fortier, 2011]. L'observation des espèces réalisée par des bénévoles et inscrite dans un territoire et des rapports sociaux coexiste avec une conception de la donnée conçue comme une information objective, définie de manière conventionnelle et facilement mobilisable grâce à des banques de données. Cette seconde conception fait entrer la nature dans le monde des procédures, des normes et de l'abstraction. Elle apparaît dans un contexte de réforme de l'État qui modifie de façon substantielle les rapports entre les associations et les pouvoirs publics.

Le 12 janvier 2007, à l'occasion du $7^{\mathrm{e}}$ Congrès français de la Nature, la ministre de l'Environnement Nelly Olin annonce :

« Je souhaite voir bientôt s'épanouir un monde associatif plus fort, moins dépendant de l'argent public, mieux reconnu par le reste de la société et toujours plus entendu. »

Cette déclaration marque un changement dans le domaine de l'action publique, qui trouve son origine, dès les années 1980, dans la décentralisation mais aussi dans les réformes de l'État d'inspiration néolibérale, à travers ce qu'il est convenu d'appeler le New public management. L'utilisation « rationnelle» des finances publiques s'accompagne en effet de la mise en place de procédures de plus en plus contraignantes, comme le suggèrent ces propos d'un directeur d'association :

\footnotetext{
«L'association a quarante ans [...], il y a un potentiel historique de connaissances dans le champ de la protection de la nature qu'on est les seuls à avoir. Donc vous voyez l'importance de ce champ de liberté dont on a joui jusqu'à présent, qui nous permettait d'espérer des financements publics quand notre intérêt et celui des pouvoirs publics se rencontraient. Ce cadre-là, depuis 2008, s'est sacrément refermé, compressé. On continue à avoir des financements, mais qu'est-ce qu'il faut batailler » (entretien, 2011).
} 
L'intégration des associations dans le champ de la commande publique en $2001^{4}$, l'adoption de la loi organique relative aux lois de finance (Lolf) la même année ${ }^{5}$ et le développement de la contractualisation ${ }^{6}$ concourent à l'affirmation d'une logique de commande publique au détriment du régime de la subvention qui a longtemps prévalu dans les rapports entre l'État et les associations ${ }^{7}$. En d'autres termes, ce sont désormais les pouvoirs publics qui définissent les besoins et le contenu de la prestation et non plus les associations qui sont à l'initiative des projets. Ce qui fait dire au responsable de l'association cité plus haut :

« On nous prend pour des cabinets d'études [...]. Ils [les pouvoirs publics] nous contraignent à glisser progressivement dans un champ commercial et nous prennent pour des exécutants. »

Le recours à la procédure d'appel d'offres est de plus en plus fréquent de la part de collectivités locales, enclines à considérer les associations comme des prestataires et à les mettre en concurrence pour mener à bien les politiques dont elles ont désormais la responsabilité. Ces réformes, qui mettent de plus en plus à contribution les associations pour réaliser des missions relevant auparavant de l'administration, se conjuguent, comme nous allons le voir à présent, à une injonction croissante à la professionnalisation du monde associatif par les pouvoirs publics.

\section{Les associations entre bénévolat et professionnalisation}

La professionnalisation des activités au sein des associations de protection de la nature se développe dans le courant des années 1990 et concerne en priorité les grandes associations [Micoud, 2001]. Aux yeux de certains observateurs, le recours au dispositif « emplois jeunes », en 1997, marque le début de ce mouvement [Dussuet, Flahault, 2008]. Dans les faits, la professionnalisation emprunte des voies multiples qui peuvent être rapportées, dans le cas des associations productrices de données, à la conjonction

4. Liée à la réforme du code des marchés publics.

5. Celle-ci tend à substituer à une logique de moyens une logique de résultats.

6. Destinée à garantir la transparence dans l'usage des fonds publics.

7. Cette évolution n'est pas spécifique aux associations environnementales mais concerne le monde associatif dans son ensemble [Hely, 2001]. 
de plusieurs phénomènes. Au développement d'un marché de la connaissance tout d'abord : avec la multiplication des politiques de conservation de la biodiversité, on assiste à une remise en cause du quasi-monopole de l'expertise des associations au profit de bureaux d'études spécialisés et d'institutions para-publiques (Conservatoires botaniques, Conservatoires régionaux d'espaces naturels) faisant appel à des salariés qualifiés. La professionnalisation apparaît alors, aux yeux d'une fraction des associations, comme un moyen de conserver une légitimité. Le processus de rationalisation des connaissances constitue également un élément clé de cette dynamique; le développement de la micro-informatique et la mise en place de systèmes d'information nécessitent en effet de faire appel à des salariés qualifiés. Enfin, les injonctions répétées des pouvoirs publics envers les associations d'accélérer leur modernisation et de se comporter en opérateurs compétitifs sur le marché participent elles aussi de ce mouvement. Néanmoins, la professionnalisation suscite des débats et des tensions parfois vives au sein du monde associatif.

L'embauche de salariés, même en nombre limité, pose la question du sens de la participation bénévole.

«Les gens sont très conscients de leur activité en tant que bénévoles - constate ce responsable d'association. C'est quelque chose dont on est assez fier finalement. On met en avant le bénévolat. On prend du plaisir à titre individuel, on enrichit sa connaissance, c'est un acquis personnel. Mais dès qu'on fait remonter l'info [...] pas mal de gens identifient que ces données sont utilisées et font l'objet d'échanges financés, alors que c'est bien eux qui sont à la source » (entretien, 2006).

Comme le suggère Prouteau [2003], si le bénévolat demeure un engagement au service d'une cause, il devient, dans ce nouveau contexte, une ressource en main-d'œuvre dans un processus de production générateur de tensions internes. C'est ainsi que l'embauche de salariés au sein d'une association d'envergure régionale a suscité de vifs débats au sein du conseil d'administration. Des voix se sont élevées pour dénoncer :

«La présence de l'argent qui transforme une association de bénévoles en ONG avec ses employés et ses employeurs : on ne défend plus la nature, on ne l'observe plus pour essayer de comprendre comment elle fonctionne et essayer de la protéger du mieux que l'on peut, mais on l'observe pour maintenir le travail des employés de l'association » (entretien, 2007). 
On le voit, la professionnalisation est à l'origine de transformations importantes dans bon nombre d'associations [Alphandéry, Fortier, 2014 à paraître]. Cependant, ce processus est loin d'être uniforme. Chaque association, en fonction de son histoire, de ses dynamiques internes, des valeurs qu'elle met en avant est amenée à prendre position vis-à-vis de la marchandisation de ses activités, de ses partenaires publics et privés et à faire face aux tensions liées à l'embauche de salariés et à la rationalisation de ses activités. Le processus de professionnalisation au sein du monde associatif présente donc plusieurs visages que l'on peut situer sur un continuum allant des associations qui recourent à un fonctionnement fondé exclusivement sur le bénévolat à celles qui privilégient le recours à quelques salariés permanents (moins de cinq) ou en plus grand nombre (dix et au-delà), s'apparentant alors, dans ce dernier cas, à des « entreprises associatives ». Dans le contexte actuel de professionnalisation des structures, de rationalisation de la production des données et des finances publiques, toute la difficulté, pour les associations naturalistes qui mêlent dans des proportions plus ou moins fortes bénévoles et salariés ou encore activités marchandes et non-marchandes consiste à maintenir l'équilibre entre, d'un côté, ce qui fait la raison d'être de l'association et, de l'autre, les moyens qu'elle met en œuvre pour assurer son fonctionnement [Enjolras, 1998 ; Laville et al., 2001].

\section{Conclusion}

Le terme de donnée, s'il questionne plus d'une oreille maussienne, est ici lié à l'univers du calcul et de la transmission d'informations. Il s'est substitué à la notion d'observation avec la diffusion de l'informatique au sein du monde naturaliste dans le courant des années 1970. Toutefois, la dimension du don est présente dans l'engagement bénévole des amateurs et des professionnels, souvent prêts à s'investir sans compter dans le travail de collecte de données. Ces dernières apparaissent donc comme un objet complexe caractérisé par une tension permanente entre le calcul et la rationalisation d'un côté, l'univers du don de soi et des rapports de sociabilité de l'autre. Cette combinaison de la dimension scientifique avec l'expérience issue de la pluralité des attaches, qu'elles soient locales ou personnelles, constitue 
une caractéristique essentielle dans la dynamique de production des données. Ce qui est en jeu est à rapprocher de l'opposition dessinée par Manuel Castells [1999, p. 155-156] entre un « espace des lieux », qui privilégie l'interaction sociale et l'organisation politique et dont il rappelle l'importance anthropologique, et l' « espace des flux » dominant, car organisant à distance les pratiques sociales par la maîtrise des systèmes de télécommunication et d'information. L'espace des lieux représente l'épaisseur humaine et historique de la donnée : en particulier, l'ensemble des connaissances et des compétences acquises par l'expérience et mobilisées par les naturalistes, même si ces dernières relèvent du domaine du non-visible [Tsoukas, 1997 ; Strathern, 2000]. À l'inverse, la donnée envisagée dans l'espace des flux est décontextualisée, désencastrée des relations sociales, autrement dit débarrassée d'un ensemble de considérations au profit de normes susceptibles de la rendre cumulable et comparable avec d'autres données.

Il n'est pas surprenant, dans ces conditions, de constater les réticences et les oppositions manifestées par le monde associatif vis-à-vis d'un système d'information qui exige, au nom du principe de transparence, la fourniture de données élémentaires (observations brutes effectuées par les naturalistes) censées pouvoir être utilisées par d'autres. Les associations estiment en effet être plus à même d'interpréter les données qu'elles ont produites elles-mêmes, en faisant valoir leur capacité d'expertise liée à la connaissance des lieux, à leur inscription dans une histoire et à des échanges nourris entre leurs membres, tout en valorisant le travail accompli par les collecteurs de données à titre bénévole. La reconnaissance est une dimension essentielle dans cet univers non marchand, où le don et l'échange sont privilégiés. D'une manière générale, plus on assimile la nature-biodiversité à ses seules dimensions techno-scientifiques et à une hypothétique valeur instituée par un marché fictif, plus s'énoncent des approches de la nature mettant en avant des attachements, des organisations collectives, des droits historiques, des patrimoines, des identités, des générations.

Les travaux réalisés sur le développement des infrastructures destinées à permettre l'accès et le partage des données [Tsoukas, 1997 ; Hine, 2006 ; Millerand et Bowker, 2011] ont montré les limites qu'elles comportent. L'un des risques encouru est le formatage des données par les scientifiques ou les gestionnaires à 
partir de conventions, de référentiels et de protocoles discutables mais repris sans discussion, comme le suggère Geoffray Bowker :

« If we are only saving what we are counting, and if our counts are skewed in many different ways, then we are creating a new world in which those counts become more and more normalised » [Bowker, 2000, p. 675].

D'un point de vue plus pragmatique, le développement de ce type d'infrastructures (banques de données, systèmes d'information) a également des effets sur certains bénévoles qui ne se reconnaissent pas dans des procédures toujours plus contraignantes, exigeantes, au point de renoncer à fournir leurs données aux associations.

Plus fondamentalement, on peut se poser la question du devenir des associations dans ces temps de techniques de gestion triomphantes et de néolibéralisme étendant toujours plus loin la globalisation. Celles-ci sont-elles vouées à être de plus en plus mises à contribution, voire instrumentalisées par les pouvoirs publics au point d'être réduites à devenir des fournisseurs de données ? Ou vont-elles trouver les ressources nécessaires pour faire coexister des logiques d'action en tension? Comme le souligne Lionel Prouteau :

«Ce qui rend la situation de l'association spécifique, c'est qu'elle fait coexister une raison communicationnelle seule source de sens et une raison instrumentale qui devrait rester seconde, subsumée sous la première, mais qui menace de prendre son autonomie. C'est pourquoi l'association gagne à être pensée comme un espace en tension » [Prouteau, 2004, avant propos non paginé].

Or en matière de nature et, plus encore, de risques concernant la biodiversité, la plupart des associations mettent plus volontiers en avant la légitimité scientifique, qui leur semble plus facilement recevable localement, que la légitimité politique qui consiste, dans le cas présent, à faire le lien entre les questions naturalistes et les questions sociales à travers des espaces de débat. Au moment où l'on vante la souplesse des réseaux et leur efficacité, la présence associative durable sur un territoire lui permet d'exercer une vigilance, de formuler publiquement des problèmes, de lancer des alertes, bref de témoigner, avec les singularités de chacune, de son attention envers la nature. C'est, nous semble-t-il, en assumant une posture politique qu'on la qualifie de bien commun, de care ou d'intérêt général que les associations naturalistes parviendront à conserver leur spécificité. 


\section{Références bibliographiques}

Alphandéry P., Bitoun P., Dupont Y., 1991, L'Équivoque écologique, La Découverte, Paris.

Alphandéry P., Fortier A., 2011, « Les associations dans le processus de rationalisation des données naturalistes », Natures, Sciences, Sociétés, 19, p. 22-30.

Alphandéry P., Fortier A., Sourdril A., 2012, « Les données entre normalisation et territoire : la construction de la trame verte et bleue », Développement durable et territoires, vol. $3, \mathrm{n}^{\circ} 2$, juil., <http://developpementdurable. revues.org/9282>, 12 juillet 2012 .

Blandin P., 2009, De la protection de la nature au pilotage de la biodiversité, Quae, Paris.

BowKER Geoffrey, C., 2000, « Biodiversity datadiversity », Social Studies of Science, 30 (5), p. 643-683.

Brahy N., LouAfI S., 2004, La Convention sur la biodiversité à la croisée de quatre discours, Les rapports de l'IDDRI, $\mathrm{n}^{\circ} 3$.

Callon M., Lascoumes P., Barthes Y., 2001, Agir dans un monde incertain, Seuil, Paris.

CAstells M. 1999, L'Ère de l'information. I. La Société en réseau, Fayard, Paris.

Chapoulie J. M., 1973, « Sur l'analyse sociologique des groupes professionnels ». Revue française de sociologie, XIV, p. 86-114.

Delaporte Y., 1984, « Des insectes et des hommes », Les Temps modernes, 450 , p. $1235-1263$.

Demazière D., 2009, « Professionnalisations problématiques et problématiques de la professionnalisation », Formation Emploi, 108, p. 83-90.

Dupré L., Micoud A., 2007, « Savoirs publics sur la nature et politiques publiques de l'environnement : rôle et place des naturalistes amateurs et des professionnels », in Charvolin F., Micoud A., Nyhart L. K. (dir.), Des sciences citoyennes? La question de l'amateur dans les sciences naturalistes, éd. de L'Aube, La Tour d'Aigues.

Dussuet A., Flahault E., 2008, «Professionnalisation, qualification, reconnaissance : une articulation délicate dans le monde associatif », Nantes, 29-30 sept. (document en ligne).

DURAND-BOURLIER M., 2003, «Quels réseaux d'information pour une meilleure prise en compte de la biodiversité dans les politiques publiques? », Rapport pour le conseil régional du Nord-Pas-de-Calais.

Ellis R., Grove-White R., Vogel J., Waterton C., 2005, Nature : Who Knows?, Natural History Museum Publication, «English Nature », Lancaster University. 
EnJolras B., 1998, « Crise de l'État-providence, lien social et associations : éléments pour une socioéconomie critique », Revue du MAUSS semestrielle, « Une seule solution, l'association? Socio-économie du fait associatif », 11, p. 223-236.

Hely M., 2011, «Quelle professionnalisation pour le monde associatif ? Entretien par Yves Lochard, Nadège Vezinat et Arnaud Trenta », <www. laviedesidees.fr>, 25 nov.

Hine C., 2006, « Databases as scientific instruments and their role in the ordering of scientific work », Social Studies of Science, vol. 36, 2, p. 269-298.

Lascoumes P., 1994, L'Éco-pouvoir, La Découverte, Paris.

Laville J.-L., Caillé A., Chanial P., Dacheux É., Eme B., Latouche S., 2001, Associations, démocratie et société civile, La Découverte/MAUSS/CRIDA, Paris.

Lawrence A., Turnhout E., 2010, «Personal meaning in the public sphere : the standardisation and rationalisation of biodiversity data in the UK and the Netherlands », Journal of rural studies, 26, p. 353-360.

Matagne P., 1999, Aux origines de l'écologie, CTHS, Paris.

MAURIN H., 1997, « Les missions du service du patrimoine naturel (IEGB/ $\mathrm{MNHN})$ : connaissance et suivi du patrimoine naturel, espèces et espaces », communication au colloque « Espaces naturels terrestres : de la décision à l'action », AICEF, supplément $n^{\circ} 35$ de Forestier, oct., Orléans.

Micoud A., 2001, « La nébuleuse associative », in L'Environnement, question sociale, O. Jacob, Paris, p. 119-129.

Millerand F., Bowker G. C., 2008, "Metadata, trajectoires et « énaction », in Rosental C. (dir.), La Cognition au prisme des sciences sociales, éd. des Archives Contemporaines, Paris. p. 277-303.

Mormont M., 1998, « Environnement et jeux transactionnels », in Aubert F., Sylvestre J.-P. (dir.) Écologie et société, p. 43-60.

Moscovici S., 2002, De la nature, Metaillée, Paris.

Pestre D., 2013, À contre-science, Seuil, Paris.

Prouteau L., 2003, « Des associations sous tensions », in Prouteau L. (dir.), Les Associations entre bénévolat et logique d'entreprise, Presses Universitaires de Rennes, p. 7-26.

RAFFin J-P., Ricou G., 1985, « Le lien entre les scientifiques et les associations de protection de la nature : approche historique », Protection de la nature, L'Harmattan, Paris, p. 61-73.

RÉmY E., 1995, « L'élaboration d'un savoir naturaliste », Études rurales, 137, p. 55-72.

Stratjern M., 2000, « The tyranny of transparency », British Educational Research Journal, vol. 26, 3, p. 309-321. 
Tsouka H., 1997, « The tyranny of light : the temptations and paradoxes of information society », Futures, 29, p. 827-843.

Wittorsky R., 2008, « La professionnalisation », Savoirs, 17, p. 9-36. 